\title{
Magnolin Protects against Contrast-Induced Nephropathy in Rats via Antioxidation and Antiapoptosis
}

\author{
Feng Wang, ${ }^{1}$ Guangyuan Zhang, ${ }^{2}$ Yang Zhou, ${ }^{3}$ Dingkun Gui, ${ }^{1}$ \\ Junhui Li, ${ }^{1}$ Tao Xing, ${ }^{4}$ and Niansong Wang ${ }^{1}$ \\ ${ }^{1}$ Department of Nephrology, Shanghai Jiao Tong University Affiliated Sixth People’s Hospital, 600 Yishan Road, \\ Shanghai 200233, China \\ ${ }^{2}$ Department of Urology, Shanghai Jiao Tong University Affiliated First People's Hospital, 100 Haining Road, Shanghai 200080, China \\ ${ }^{3}$ Department of Nephrology, The First Affiliated Hospital of Harbin Medical University, No. 23 Youzheng Street, Nangang District, \\ Harbin 150001, China \\ ${ }^{4}$ Florey Institute of Neuroscience and Mental Health, University of Melbourne, VIC 3010, Australia
}

Correspondence should be addressed to Feng Wang; zyzwq1030@gmail.com

Received 2 August 2014; Accepted 1 October 2014; Published 21 October 2014

Academic Editor: Daniela Giustarini

Copyright (C) 2014 Feng Wang et al. This is an open access article distributed under the Creative Commons Attribution License, which permits unrestricted use, distribution, and reproduction in any medium, provided the original work is properly cited.

Background. Magnolin is the major active ingredient of the herb Magnolia fargesii which has anti-inflammatory and antioxidative effects. Oxidative stress and apoptosis are involved in the pathogenesis of contrast-induced nephropathy (CIN). We hypothesize that Magnolin could protect against CIN through antioxidative and antiapoptotic properties. Methods. To test whether Magnolin could attenuate CIN, oxidative stress and apoptosis, in vivo and in vitro, we utilized a rat model of ioversol-induced CIN and a cell model of oxidative stress in which HK2 cells were treated with $\mathrm{H}_{2} \mathrm{O}_{2}$. Rats were assigned to 4 groups ( $n=6$ per group): control group, ioversol group (ioversol-induced CIN), vehicle group (CIN rats pretreated with vehicle), and Magnolin group (CIN rats pretreated with $1 \mathrm{mg} / \mathrm{kg}$ Magnolin). Results. The results showed that magnolin ameliorated the renal tubular necrosis, apoptosis, and the deterioration of renal function $(P<0.05)$. Furthermore, Magnolin reduced the renal oxidative stress, suppressed caspase-3 activity, and increased Bcl-2 expression in vivo and in vitro. Conclusion. Magnolin might protect CIN in rats through antioxidation and antiapoptosis.

\section{Introduction}

The use of contrast media is becoming more and more common in clinical diagnostic and interventional procedures $[1,2]$. As a result, contrast-induced nephropathy (CIN) is a frequent complication in clinical medical practice. The incidence of CIN varies from $3 \%$ to $14 \%$ and can reach as high as $20 \%$ in patients at high risk [3-5]. Furthermore, CIN is the third leading cause of hospital-acquired acute kidney injury, accounting for $\sim 12 \%$ of all cases of acute renal failure. CIN can result in an increased medical cost, long admission, disease deterioration, dialysis, and even death $[6,7]$. Recent evidences suggest that $\mathrm{CIN}$ can lead to long-term decline in renal function as well as mortality $[8,9]$. Thus, it is important and urgent to better understand the mechanism by which CIN occurs and to develop new preventive therapies.

The pathogenesis of CIN is primarily due to ischemia in the renal medulla resulting from intrarenal vasoconstriction, oxidative stress, and contrast media's renal toxicity. Oxidative stress plays a pivotal role in the pathophysiology of CIN which directly results in tubular damage, endothelial dysfunction, and dysfunction of tubular transport $[10,11]$. Therefore, oxidative stress attenuation is a major goal in the CIN prevention research [12]. As an antioxidant, $N$-acetylcysteine is oxygen derived free radicals scavenger, which may lead to improved endothelial dysfunction [13, 14]. Apart from reducing the contrast media volume, cautiously avoiding hypovolemia, and carefully considering contrast delivery in 
high-risk patients, antioxidant is a promising strategy for the CIN prevention.

Magnolin is an important active ingredient of Magnolia fargesii volatile oil [15]. It was reported that Magnolia fargesii and Magnolin had antioxidative, anti-inflammatory, and vasodilatory effects [16]. But it is unknown whether Magnolin could protect against CIN. We hypothesized that Magnolin might protect against CIN in rats due to antioxidant and antiapoptotic effects. The aim of this study was to investigate whether Magnolin could prevent CIN and to identify the possible mechanisms.

\section{Materials and Methods}

2.1. Magnolin and Animals. The Magnolin reagents were provided by Dongfang Pharmaceutical Company (Shanghai, China). Before use, Magnolin was solubilized in DMSO for administration. Male Sprague-Dawley rats from Shanghai Science Academy animal center, weighing $200 \pm 20$ g, were housed in individual cages under controlled conditions of light ( $12 \mathrm{~h} \mathrm{dark} / 12 \mathrm{~h}$ light cycle) and $20 \sim 23^{\circ} \mathrm{C}$. All the rats were allowed to have standard diet and tap water.

2.2. Rat CIN Model and Experimental Design. The rats were divided into control group (CTL) $(n=6)$, ioversol group (Iov) $(n=6)$, vehicle group (Veh) $(n=6)$, and Magnolin group (Mag) $(n=6)$. Rats in the Iov, Veh, and Mag groups were anesthetized with $50 \mathrm{mg} / \mathrm{kg}$ pentobarbital and given a tail vein injection of indomethacin (Sigma, USA) (10 mg/kg), followed by ioversol (Hengrui Corp., China) ( $3 \mathrm{~g} / \mathrm{kg}$ organically bound iodine) and N-nitro-L-arginine methyl ester (L-NAME) (Sigma, USA). Rats in Mag group also receive a subcutaneous injection of Magnolin $(1 \mathrm{mg} / \mathrm{kg})$ 15 minutes prior to the CIN inducing injections. Rats in vehicle group received the same volume vehicle at each time point. The rats received recovery in metabolic cages for 24 hours for the urine sample collection. After 24 hours, the left kidney was harvested for associated measurements. The right kidney was fixed in 10\% formalin for histological assessments. Blood samples were collected to isolate serum and then stored in a $-80^{\circ} \mathrm{C}$ freezer.

2.3. Biochemical Markers Determination of Blood and Urine Samples. Automatic biochemical analyzer (Hitachi 7600, Ichige, Japan) was employed to determine blood urea nitrogen (BUN), serum creatinine (SCr). Two novel markers of early stage kidney injury, urinary kidney injury molecule1 (uKIM-1), and serum neutrophil gelatinase-associated lipocalin (sNAGL) were measured using ELISA kits (R\&D, USA).

2.4. Histological Examinations. The fixed left kidney was dehydrated in ethanol and embedded in paraffin. Kidney tissue blocks were cut into $3 \mu \mathrm{m}$ sections and subjected to hematoxylin-eosin (H.E.) staining and periodic acidSchiff (PAS) staining. The histological scoring was assessed using grading tubular necrosis, loss of brush border, cast formation, and tubular dilatation in 10 randomly chosen, nonoverlapping fields. The renal injury degree was estimated by the following criteria: 0 , none; $1,0-10 \% ; 2,11-25 \% ; 3,26-$ $45 \%$; 4, 46-75\%; and 5, 76-100\%, as described previously [17].

2.5. Lipid Peroxidation/ROS Production of Renal Tissues. ROS level in kidney was assayed as previously described [18]. The nonpolar compound dihydrodichlorofluorescein diacetate (H2 DCFH-DA), after conversion to a polar derivative by intracellular esterases, can rapidly react with ROS to form the highly fluorescent compound dichlorofluorescein. The commercial available assay kit (E004, Nanjing Jiancheng Bioengineering Institute, Jiangsu, China) was used. As previously described [19], malondialdehyde (MDA) and superoxide dismutase (SOD) were detected in the kidney tissues. The content of MDA was determined by the thiobarbituric acid method (A003-1, Nanjing Jiancheng Bioengineering Institute, Jiangsu, China). The SOD activity assay kit (A001-1, Nanjing Jiancheng Bioengineering Institute, Jiangsu, China) was enrolled. SOD activity was determined by inhibition of nitroblue tetrazolium reduction due to superoxide anion generation by a xanthine-xanthine oxidase system. All the assays were carried out according to the manufacture's protocol using spectra microplate reader (model A-5082, Tecan, Australia).

2.6. Assessment of Renal Apoptosis in CIN Rats. A TUNEL staining for cell apoptosis in renal tissues was employed to evaluate the apoptosis extent following the instructions of the manufacturer (Roche Diagnostics, Mannheim, Germany).

2.7. Caspase-3 Activity and Bcl-2 Expression in Renal Tissues. The caspase- 3 activity in renal tissues was measured using a commercial kit (Beyotime, Nantong, China). Western blot was employed to assess the $\mathrm{Bcl}-2$ expression in renal tissues with an anti-Bcl-2 polyclonal antibody (\#sc-492, Santa Cruz, USA) (dilution 1:200) as described previously [20].

2.8. An Oxidative Stress Model of HK2 Cells In Vitro. HK2 cells (ATCC, Manassas, Virginia) were cultured in K-SFM at $37^{\circ} \mathrm{C} 5 \% \mathrm{CO}_{2}$, supplemented with $5 \mathrm{ng} / \mathrm{mL}$ human recombinant EGF and $0.05 \mu \mathrm{g} / \mathrm{mL}$ bovine pituitary extract. HK2 cells were preconditioned with Magnolin $(10 \mu \mathrm{g} / \mathrm{mL}$ and $40 \mu \mathrm{g} / \mathrm{mL}$, resp.) followed by $500 \mu \mathrm{mol} / \mathrm{L} \mathrm{H}_{2} \mathrm{O}_{2}$ to induce oxidative stress injury. To investigate the antioxidative and antiapoptotic effects of Magnolin, caspase-3 activity (Beyotime, Nantong, China) and intracellular ROS (Cell Biolabs, San Diego, USA) were measured with commercial kits. In addition, Bcl-2 expression was determined using Western blot.

2.9. Statistical Analysis. The statistical software SPSS (Ver. 18.0) was used for data analysis. One-way ANOVA with Sidak post hoc test or Kruskal-Wallis with Dunn's post test was employed to determine the differences in groups. A value of $P<0.05$ was considered significant. 


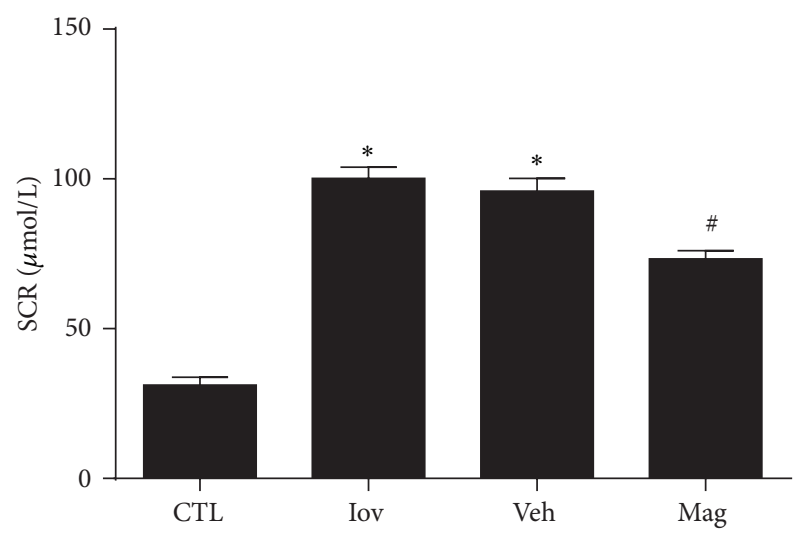

(a)

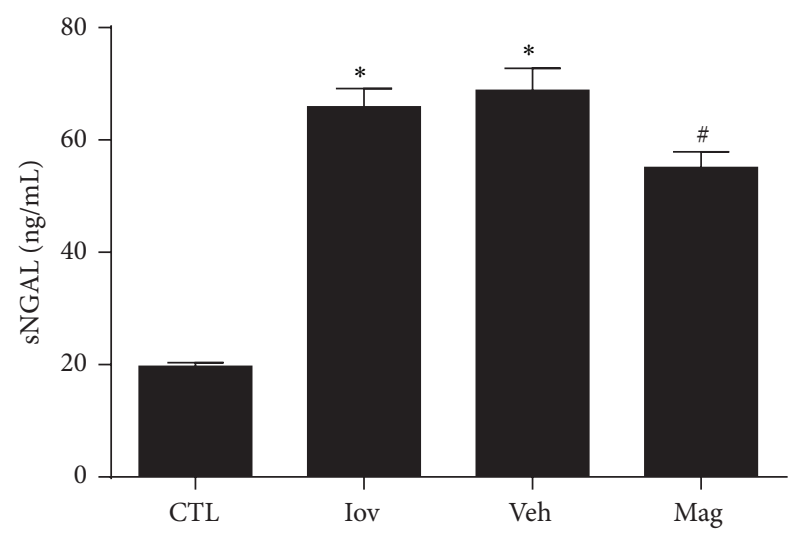

(c)

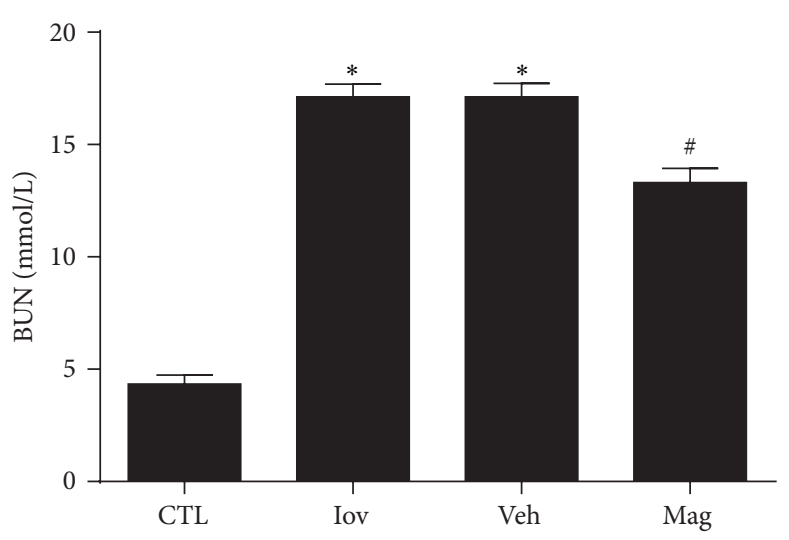

(b)

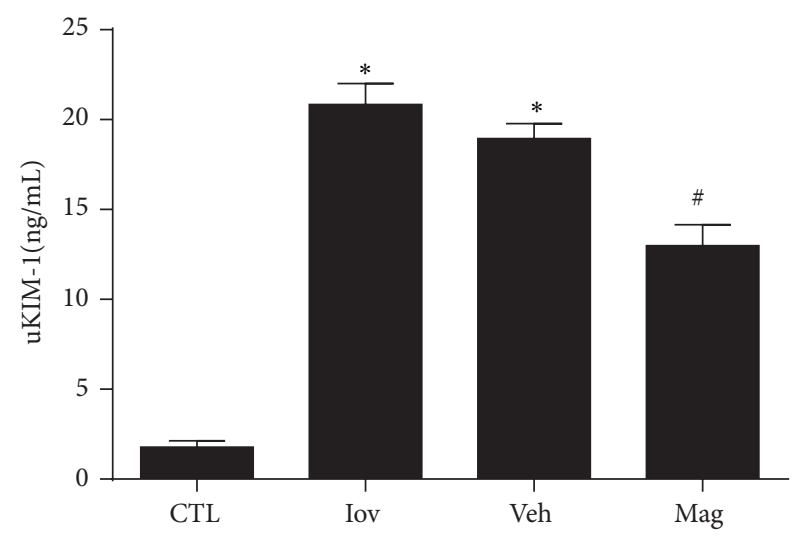

(d)

FIGURE 1: Magnolin decreased levels of serum creatinine (a), blood nitrogen urea (b), serum NGAL (c), and urinary KIM-1 (d) in CIN rats. ${ }^{*} P<0.01$, versus CTL group. ${ }^{\#} P<0.01$, versus Veh group.

\section{Results}

3.1. Magnolin Decreased Levels of SCr, BUN, sNGAL, and uKIM-1 in CIN Rats. Animals subjected to CIN in Iov and Veh groups presented significantly increased levels of SCr, BUN, sNGAL, and uKIM-1 compared with CTL group. However, SCr, BUN, sNGAL, and uKIM-1 levels were all decreased in rats treated with Magnolin as shown in Figure 1. Thus, Magnolin can attenuate the levels of renal function parameters.

3.2. Magnolin Ameliorated Renal Histological Damage. The tubular detachment, foamy degeneration, and necrosis can be seen in rats of Iov and Veh groups. Moreover, the most pronounced and severe alterations were observed in the corticomedullary boundary area. The morphological alterations in Iov and Veh groups were much more severe than that in Mag group. However, tubular scores in Mag group were ameliorated with the Magnolin treatment (Figure 2), which was consistent with Figure 1.

3.3. Magnolin Reduced Oxidative Stress in Renal Tissues. Elevated renal MDA and decreased renal SOD were observed in Iov and Veh groups compared with CTL group $(P<$ 0.01 ) (Figures 5(a) and 5(b)). However, pretreatment with Magnolin reduced renal MDA levels and increased renal SOD levels dramatically. Thus, the results demonstrated that Magnolin might reduce oxidative stress from both directions in CIN rats.

3.4. Magnolin Inhibited Renal Apoptosis. TUNEL assay revealed that the TUNEL-positive cells were much less in Mag group than that in Iov and Veh groups as shown in Figure 3. In comparison, Magnolin preconditioning reduced the tubular apoptosis in rats with ioversol-induced nephropathy.

3.5. Magnolin Decreased Renal Caspase-3 Activity and Increased Bcl-2 Expression in CIN. To further determine whether Magnolin took protective effects on cell apoptosis, caspase- 3 activity and Bcl-2 expression were measured. The results showed rats in Iov group and Veh group had significantly higher caspase-3 activity and lower Bcl-2 levels than that in CTL group (both $P<0.01$ ). Furthermore, rats in Mag group exhibited decreased caspase- 3 activity and increased Bcl-2 $(P<0.05)$ (Figure 4$)$, which was consistent with Figure 3. 


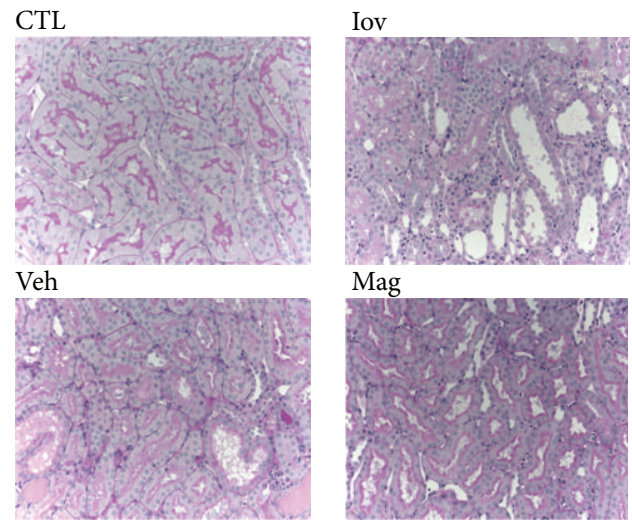

(a)

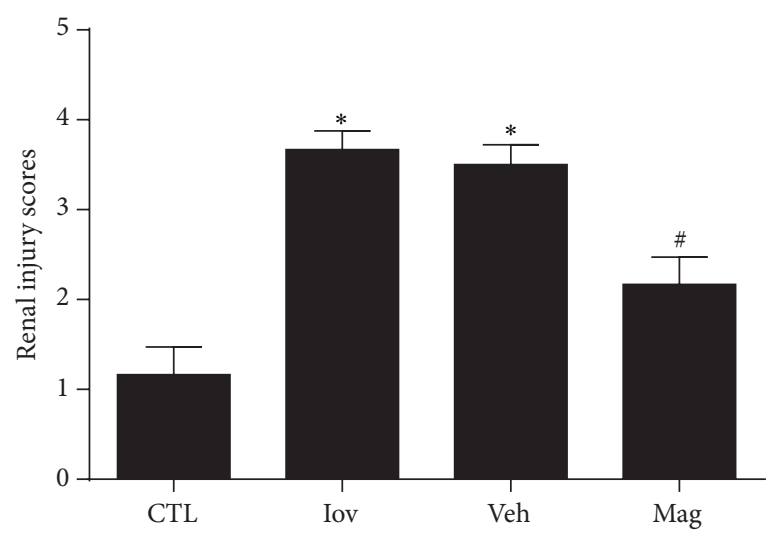

(b)

FIGURE 2: Magnolin ameliorated renal histological damage. (a) Representative renal sections from control, ioversol, vehicle, and Magnolin groups (PAS, 400x). (b) Renal injury scoring and quantitative analysis. ${ }^{*} P<0.01$, versus CTL group. ${ }^{\#} P<0.05$, versus Veh group.

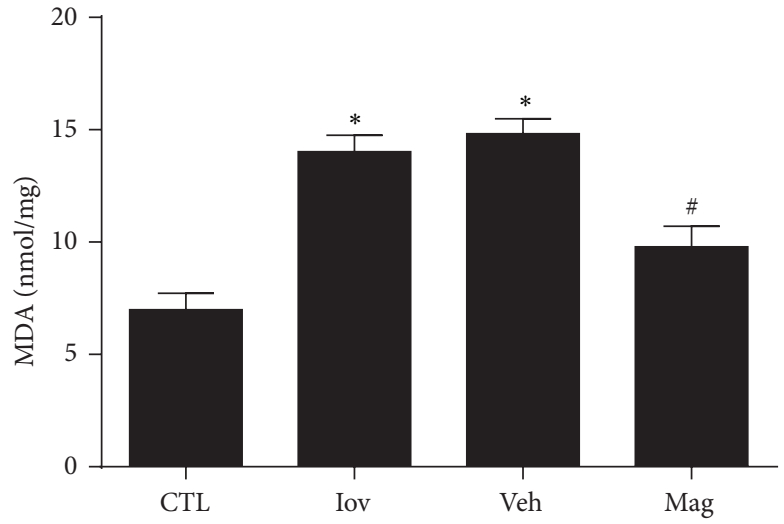

(a)

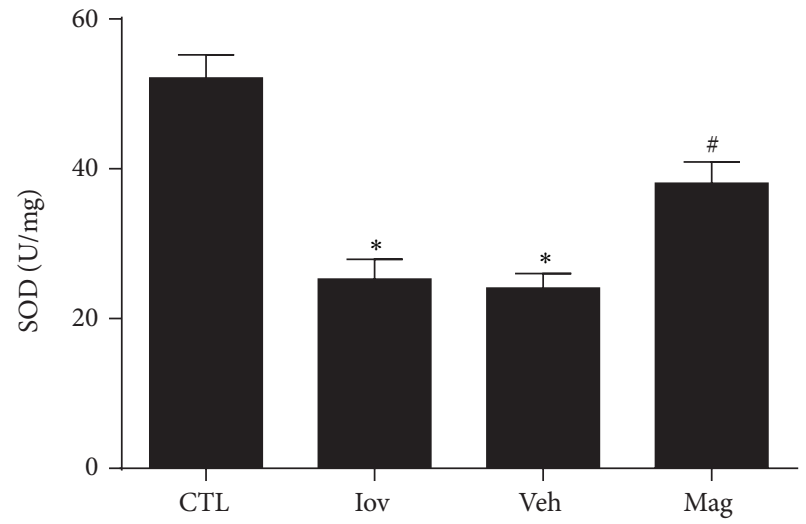

(b)

FIgURE 3: Magnolin reduced oxidative stress in renal tissues. (a) Renal MDA levels in rats from control, ioversol, vehicle, and Magnolin groups. (b) Renal SOD levels in rats from control, ioversol, vehicle, and Magnolin groups. ${ }^{*} P<0.01$, versus CTL group. ${ }^{\#} P<0.05$, versus Veh group.
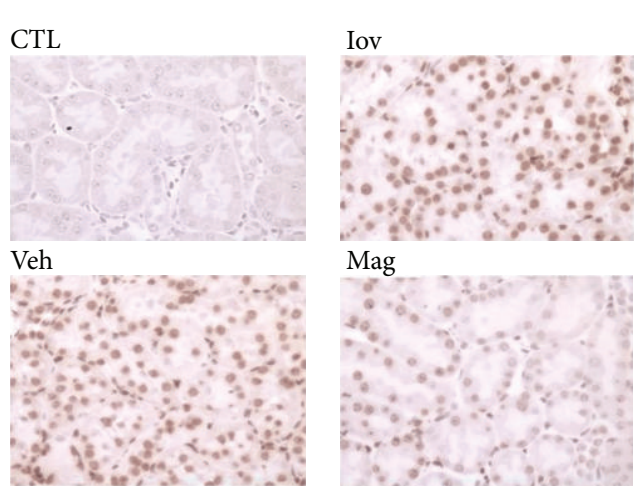

(a)

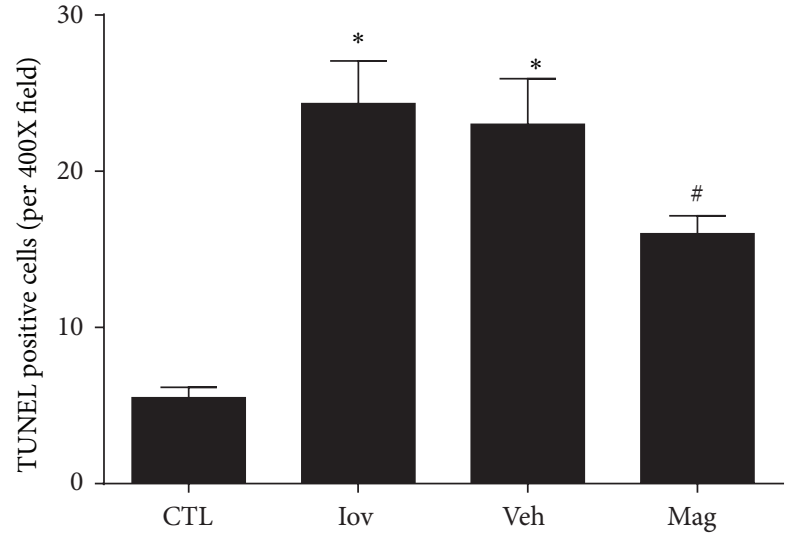

(b)

FIGURE 4: Magnolin inhibited renal apoptosis. (a) Representative renal sections from control, ioversol, vehicle, and Magnolin groups (TUNEL assay, 400x). (b) Quantitative analysis of tubular cells apoptosis. ${ }^{*} P<0.01$, versus CTL group. ${ }^{\#} P<0.05$, versus Veh group. 


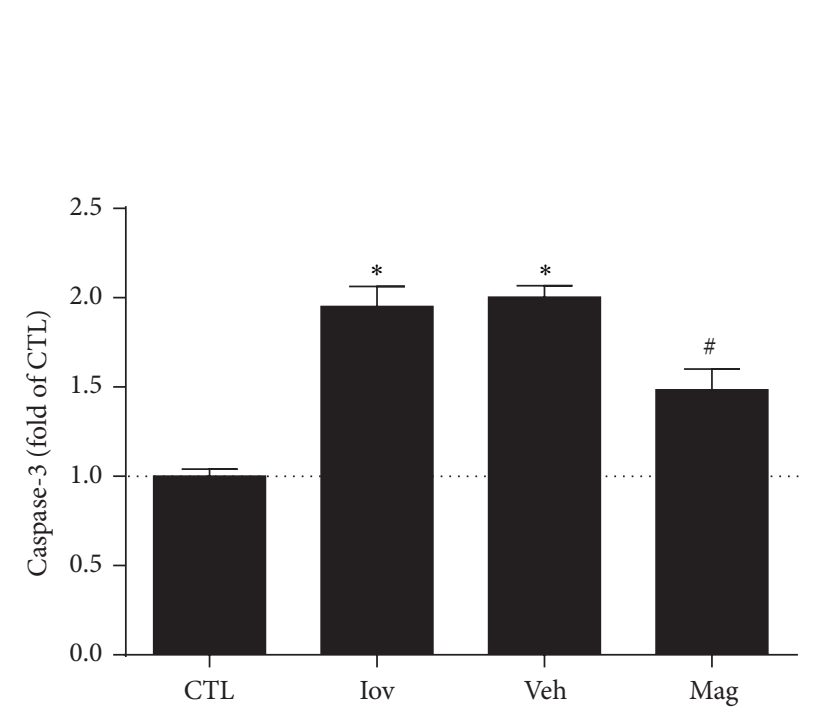

(a)
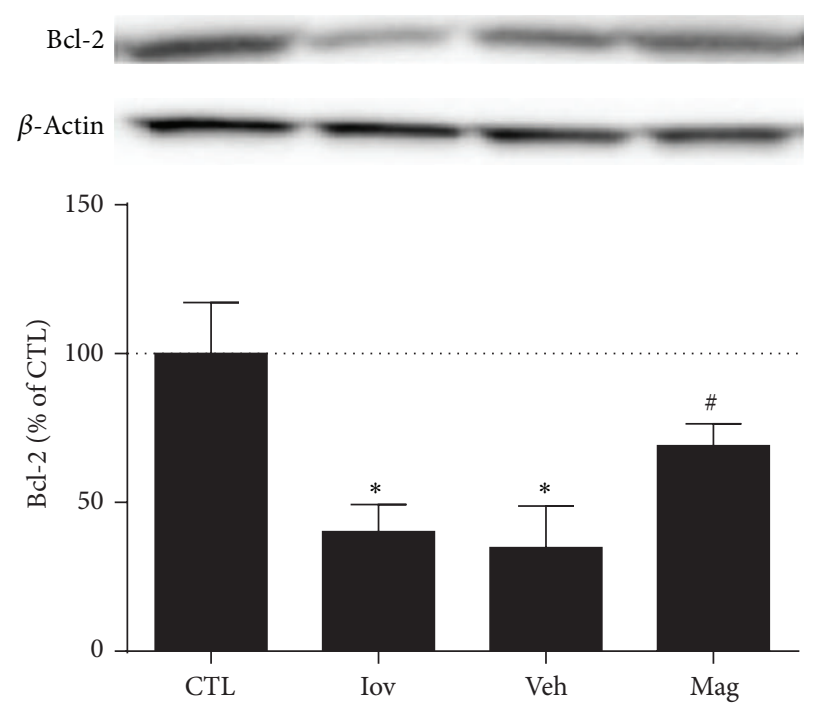

(b)

FIGURE 5: Magnolin decreased renal caspase-3 activity and increased Bcl-2 expression in CIN. (a) Renal caspase-3 activity in rats from control, ioversol, vehicle, and Magnolin groups. (b) Renal Bcl-2 expression (Western blot) in rats and quantitative analysis from control, ioversol, vehicle, and Magnolin groups. ${ }^{*} P<0.01$, versus CTL group. ${ }^{\#} P<0.05$, versus Veh group.

3.6. Magnolin Protected against Oxidative Stress and Apoptosis in HK2 Cells. The effects of Magnolin on ROS levels, caspase3 activity, and Bcl-2 expression were examined in HK2 cells treated with $\mathrm{H}_{2} \mathrm{O}_{2}$. In contrast with control group, the ROS and caspase-3 levels elevated in $\mathrm{HK} 2$ cells treated with $\mathrm{H}_{2} \mathrm{O}_{2}$ $(500 \mu \mathrm{mol} / \mathrm{L})$, while Bcl-2 expression was attenuated $(P<$ 0.01 ). However, Magnolin (both $10 \mu \mathrm{g} / \mathrm{mL}$ and $40 \mu \mathrm{g} / \mathrm{mL}$ ) decreased the ROS levels and caspase-3 activity and increased Bcl-2 expression (Figure 6).

\section{Discussion}

There are lots of circumstances in which it is medically necessary to utilize contrast-enhanced imaging, despite a significant risk of CIN. Thus, it is urgent to find the most beneficial treatments to prevent CIN from developing [12]. The present study demonstrated for the first time that Magnolin pretreatment before the administration of contrast media might prevent the acute kidney injury in rats. CIN is a renal injury not only due to the toxicity of contrast media, but also due to renal ischemia, oxidative stress injury, and cell apoptosis [11]. According to our results, Magnolin ameliorated renal injury, tubular necrosis, and the deterioration of renal function. Furthermore, Magnolin reduced oxidative stress, suppressed caspase-3 activity, and increased Bcl-2 expression in vivo and in vitro.

Oxidative stress plays an important role in the CIN pathogenesis. The administration of contrast media elevates ROS levels that can cause lipid peroxidation. Development or identification of new drugs that can scavenge ROS has been a major focus for the prevention of CIN in highrisk patients. In this study, Magnolin preconditioned rats expressed lower renal MDA, higher renal SOD, and reduced kidney injury. In addition, Magnolin decreased ROS generation and caspase-3 activity, while it increased the Bcl-2 expression in an oxidative stress model in vitro. Therefore, Magnolin presented antioxidative effects in this CIN model, which was consistent with previous reports [21]. It is deduced that Magnolin protecting against kidney injury in CIN rats might be due to its antioxidative effects.

Apoptosis is another mechanism of acute kidney injury including CIN $[22,23]$. Suppressing apoptosis pathways may attenuate pathological alterations in CIN. It has been reported that both mitochondrial and intrinsic apoptosis pathways participated in the tubular injuries of CIN [24]. Bcl-2 is an antiapoptotic protein that can inhibit caspase-3. Increased Bcl-2 levels and decreased caspase-3 activity might contribute to reduced apoptosis [25]. In present study, we observed severe histological injury and extensive apoptosis in the CIN kidney, consistent with previous results [25, 26]. Moreover, increased caspase- 3 activity and decreased Bcl-2 expression were partly reversed by Magnolin in CIN rats or HK2 cells treated with $\mathrm{H}_{2} \mathrm{O}_{2}$. Thus, it can be speculated that Manolin's renoprotection in CIN rats might be associated with $\mathrm{Bcl}$ 2/caspase-3 antiapoptosis pathway.

Our previous data showed that Magnolin protected against diabetic nephropathy through anti-inflammatory and antiplatelet effects [27]. Combined with the present results, Magnolin exhibited antioxidative and anti-inflammatory effects in rat kidney injury models, which was consistent with previous reports [28]. However, the detailed molecular mechanism that drives this protection remains unknown. Further studies focused on the molecular mechanisms will be important for both understanding the pathogenesis of CIN and evaluating Magnolin's therapeutic potential. 


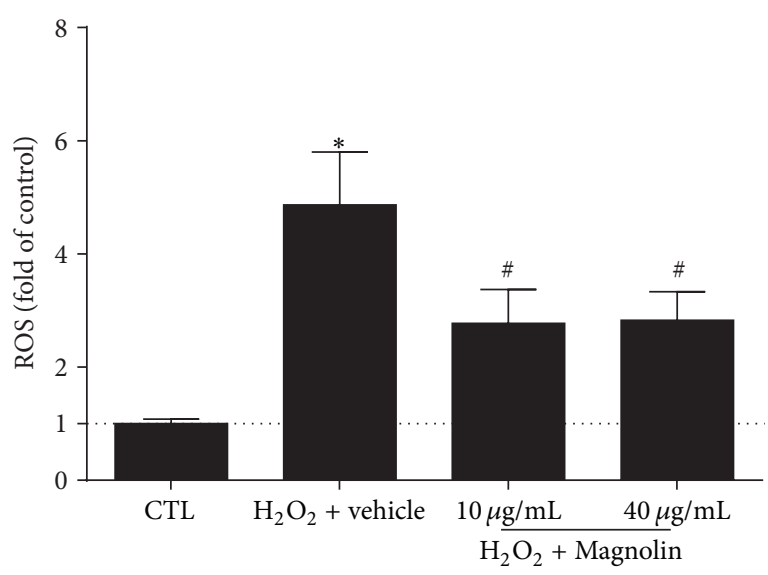

(a)

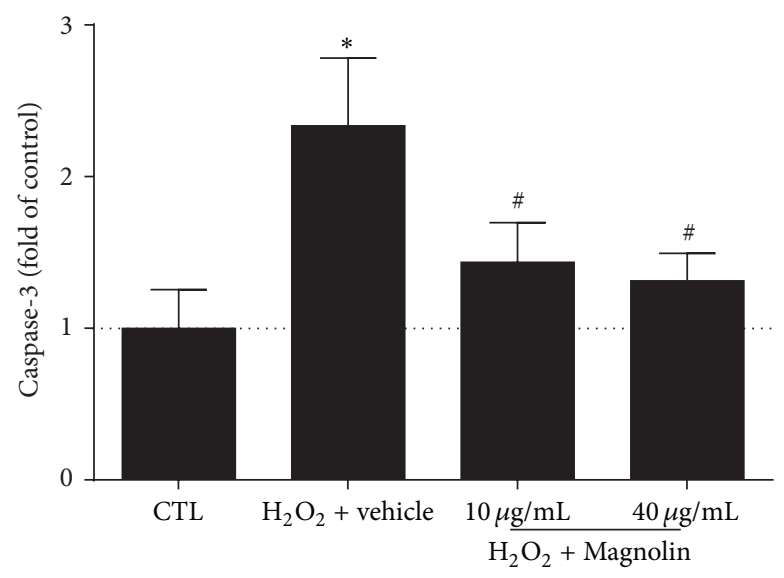

(b)

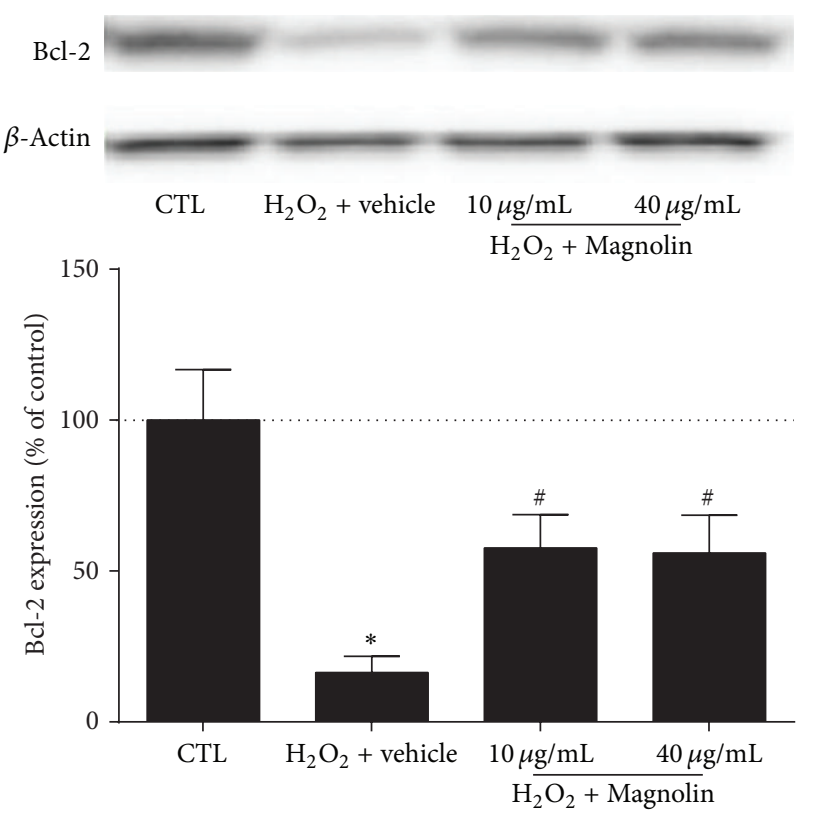

(c)

FIGURE 6: Magnolin protected against oxidative stress and apoptosis in HK2 cells. (a) The effects of Magnolin on ROS levels in HK2 cells treated with $\mathrm{H}_{2} \mathrm{O}_{2}(500 \mu \mathrm{mol} / \mathrm{L})$. (b) The effects of Magnolin on caspase-3 activity in $\mathrm{HK} 2$ cells treated with $\mathrm{H}_{2} \mathrm{O}_{2}(500 \mu \mathrm{mol} / \mathrm{L})$. (c) The effects of Magnolin on Bcl-2 expression in $\mathrm{HK} 2$ cells treated with $\mathrm{H}_{2} \mathrm{O}_{2}(500 \mu \mathrm{mol} / \mathrm{L}) .{ }^{*} P<0.01$, versus control group. ${ }^{*} P<0.05$, versus $\mathrm{H}_{2} \mathrm{O}_{2}$ group.

In conclusion, our data suggested that Magnolin exhibits renal protection in CIN model via antioxidative and antiapoptotic manners. Magnolin could be a novel antioxidant to the CIN therapy in the future.

\section{Conflict of Interests}

There is no conflict of interests for all the coauthors.

\section{Authors' Contribution}

Drs. Feng Wang, Guangyuan Zhang, and Yang Zhou contributed to this work equally.

\section{Acknowledgments}

This work was sponsored by the National Natural Science Foundation of China (81100528), the New 100-Talent Plan of Shanghai Jiao Tong University School of Medicine, and Shanghai Talents Development Fund.

\section{References}

[1] A. Asif and M. Epstein, "Prevention of radiocontrast-induced nephropathy," The American Journal of Kidney Diseases, vol. 44, no. 1, pp. 12-24, 2004.

[2] F. Wang, J. Li, B. Huang et al., "Clinical survey on contrastinduced nephropathy after coronary angiography," Renal Failure, vol. 35, no. 9, pp. 1255-1259, 2013. 
[3] M. Abe, T. Kimura, T. Morimoto, Y. Furukawa, and T. Kita, "Incidence of and risk factors for contrast-induced nephropathy after cardiac catheterization in Japanese patients," Circulation Journal, vol. 73, no. 8, pp. 1518-1522, 2009.

[4] B. A. Bartholomew, K. J. Harjai, S. Dukkipati et al., "Impact of nephropathy after percutaneous coronary intervention and a method for risk stratification," The American Journal of Cardiology, vol. 93, no. 12, pp. 1515-1519, 2004.

[5] P. J. M. Best, R. Lennon, H. H. Ting et al., "The impact of renal insufficiency on clinical outcomes in patients undergoing percutaneous coronary interventions," Journal of the American College of Cardiology, vol. 39, no. 7, pp. 1113-1119, 2002.

[6] W. F. Finn, "The clinical and renal consequences of contrastinduced nephropathy," Nephrology Dialysis Transplantation, vol. 21, no. 1, pp. i2-i10, 2006.

[7] I. Goldenberg and S. Matetzky, "Nephropathy induced by contrast media: pathogenesis, risk factors and preventive strategies," Canadian Medical Association Journal, vol. 172, no. 11, pp. 1461-1471, 2005.

[8] M. T. James, W. A. Ghali, M. L. Knudtson et al., "Associations between acute kidney injury and cardiovascular and renal outcomes after coronary angiography," Circulation, vol. 123, no. 4, pp. 409-416, 2011.

[9] M. T. James, S. M. Samuel, M. A. Manning et al., "Contrastinduced acute kidney injury and risk of adverse clinical outcomes after coronary angiography: a systematic review and meta-analysis," Circulation: Cardiovascular Interventions, vol. 6, no. 1, pp. 37-43, 2013.

[10] S. N. Heyman, S. Rosen, M. Khamaisi, J.-M. Idée, and C. Rosenberger, "Reactive oxygen species and the pathogenesis of radiocontrast-induced nephropathy," Investigative Radiology, vol. 45, no. 4, pp. 188-195, 2010.

[11] R. Solomon and H. L. Dauerman, "Contrast-induced acute kidney injury," Circulation, vol. 122, no. 23, pp. 2451-2455, 2010.

[12] N. M. Mohammed, A. Mahfouz, K. Achkar, I. M. Rafie, and R. Hajar, "Contrast-induced nephropathy," Heart Views, vol. 14, no. 3, pp. 106-116, 2013.

[13] L. F. Drager, L. Andrade, J. F. B. de Toledo, F. R. M. Laurindo, L. A. M. César, and A. C. Seguro, "Renal effects of $N$-acetylcysteine in patients at risk for contrast nephropathy: decrease in oxidant stress-mediated renal tubular injury," Nephrology Dialysis Transplantation, vol. 19, no. 7, pp. 1803-1807, 2004.

[14] S. N. Heyman, S. Rosen, and C. Rosenberger, "A role for oxidative stress," Contributions to Nephrology, vol. 174, pp. 138148, 2011.

[15] K.-S. Ahn, K. Y. Jung, J.-H. Kim, S. R. Oh, and H.-K. Lee, "Inhibitory activity of lignan components from the flower buds of Magnoliae fargesii on the expression of cell adhesion molecules," Biological and Pharmaceutical Bulletin, vol. 24, no. 9, pp. 1085-1087, 2001.

[16] J. S. Kim, J. Y. Kim, H. J. Lee et al., "Suppression of inducible nitric oxide synthase expression by furfuran lignans from flower buds of Magnolia fargesii in BV-2 microglial cells," Phytotherapy Research, vol. 24, no. 5, pp. 748-753, 2010.

[17] V. Y. Melnikov, S. Faubel, B. Siegmund, M. Scott Lucia, D. Ljubanovic, and C. L. Edelstein, "Neutrophil-independent mechanisms of caspase-1- and IL-18-mediated ischemic acute tubular necrosis in mice," Journal of Clinical Investigation, vol. 110, no. 8, pp. 1083-1091, 2002.

[18] G. Zhang, X. Zou, S. Miao et al., "The anti-oxidative role of micro-vesicles derived from human wharton-jelly mesenchymal stromal cells through NOX2/gp91(phox) suppression in alleviating renal ischemia-reperfusion injury in rats," PLoS ONE, vol. 9, no. 3, Article ID e92129, 2014.

[19] T. Wu, X.-S. Ding, W. Wang, and J. Wu, "MCI-186 (3-methyl1-phenyl-2-pyrazolin-5-one) attenuated simulated ischemia/ reperfusion injury in cultured rat hippocampal cells," Biological and Pharmaceutical Bulletin, vol. 29, no. 8, pp. 1613-1617, 2006.

[20] F. Wang, T. Xing, J. Li et al., "Renalase's expression and distribution in renal tissue and cells," PLoS ONE, vol. 7, no. 10, Article ID e46442, 2012.

[21] J. A. Baek, Y. D. Lee, C. B. Lee et al., "Extracts of Magnoliae flos inhibit inducible nitric oxide synthase via ERK in human respiratory epithelial cells," Nitric Oxide-Biology and Chemistry, vol. 20, no. 2, pp. 122-128, 2009.

[22] A. Caiazza, L. Russo, M. Sabbatini, and D. Russo, "Hemodynamic and tubular changes induced by contrast media," BioMed Research International, vol. 2014, Article ID 578974, 7 pages, 2014.

[23] A. Linkermann, F. de Zen, J. Weinberg, U. Kunzendorf, and S. Krautwald, "Programmed necrosis in acute kidney injury," Nephrology Dialysis Transplantation, vol. 27, no. 9, pp. 34123419, 2012.

[24] G. Romano, C. Briguori, C. Quintavalle et al., "Contrast agents and renal cell apoptosis," European Heart Journal, vol. 29, no. 20, pp. 2569-2576, 2008.

[25] A. I. Goodman, R. Olszanecki, L. M. Yang et al., "Heme oxygenase-1 protects against radiocontrast-induced acute kidney injury by regulating anti-apoptotic proteins," Kidney International, vol. 72, no. 8, pp. 945-953, 2007.

[26] A. Kodama, H. Watanabe, R. Tanaka et al., "A human serum albumin-thioredoxin fusion protein prevents experimental contrast-induced nephropathy," Kidney International, vol. 83, no. 3, pp. 446-454, 2013.

[27] Y. Zhou, F. Wang, L. Hao, and N. Wang, "Effects of magnoline on p-selectin's expression in diabetic rats and its reno-protection," Kidney and Blood Pressure Research, vol. 37, no. 2-3, pp. 211-220, 2013.

[28] J. Y. Kim, H. J. Lim, D. Y. Lee et al., "In vitro anti-inflammatory activity of lignans isolated from Magnolia fargesii," Bioorganic \& Medicinal Chemistry Letters, vol. 19, no. 3, pp. 937-940, 2009. 


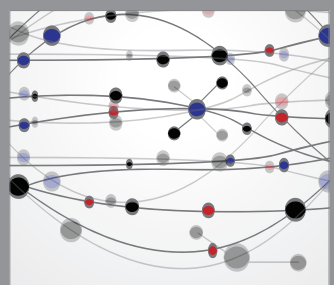

The Scientific World Journal
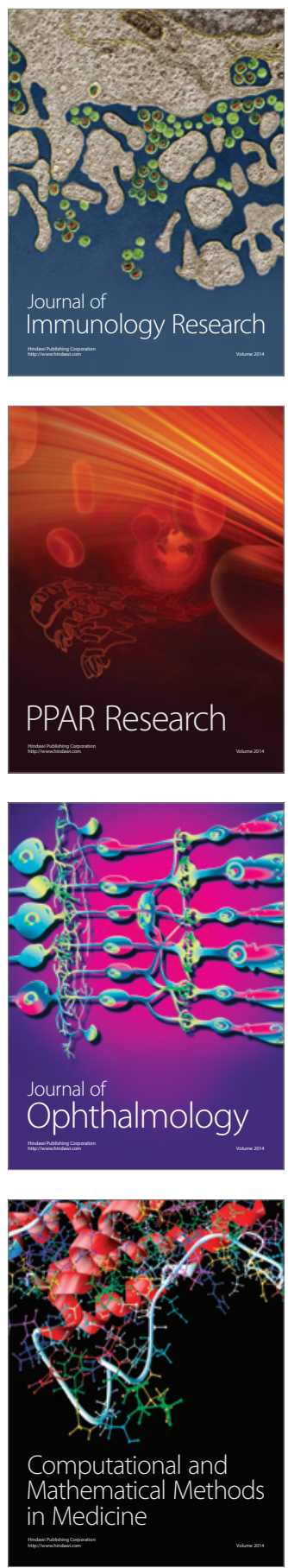

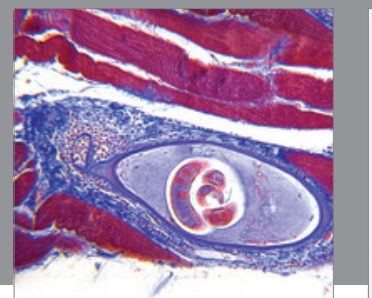

Gastroenterology

Research and Practice
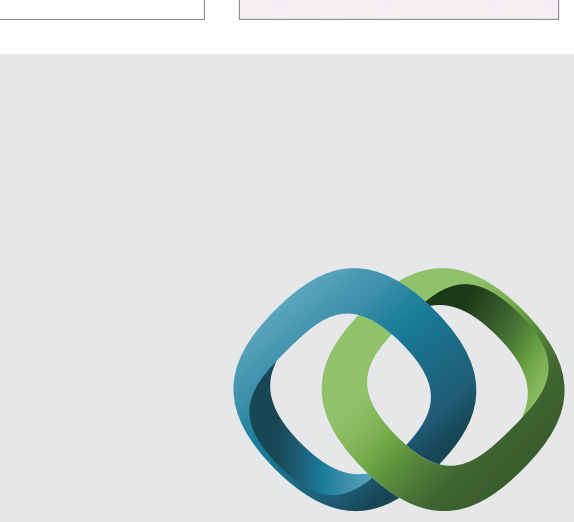

\section{Hindawi}

Submit your manuscripts at

http://www.hindawi.com
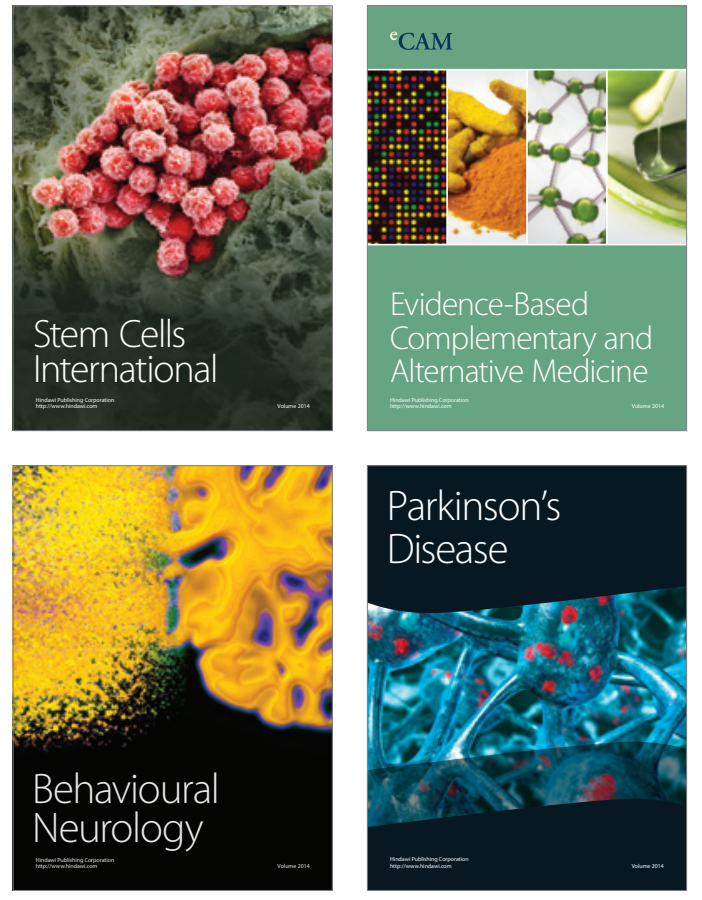
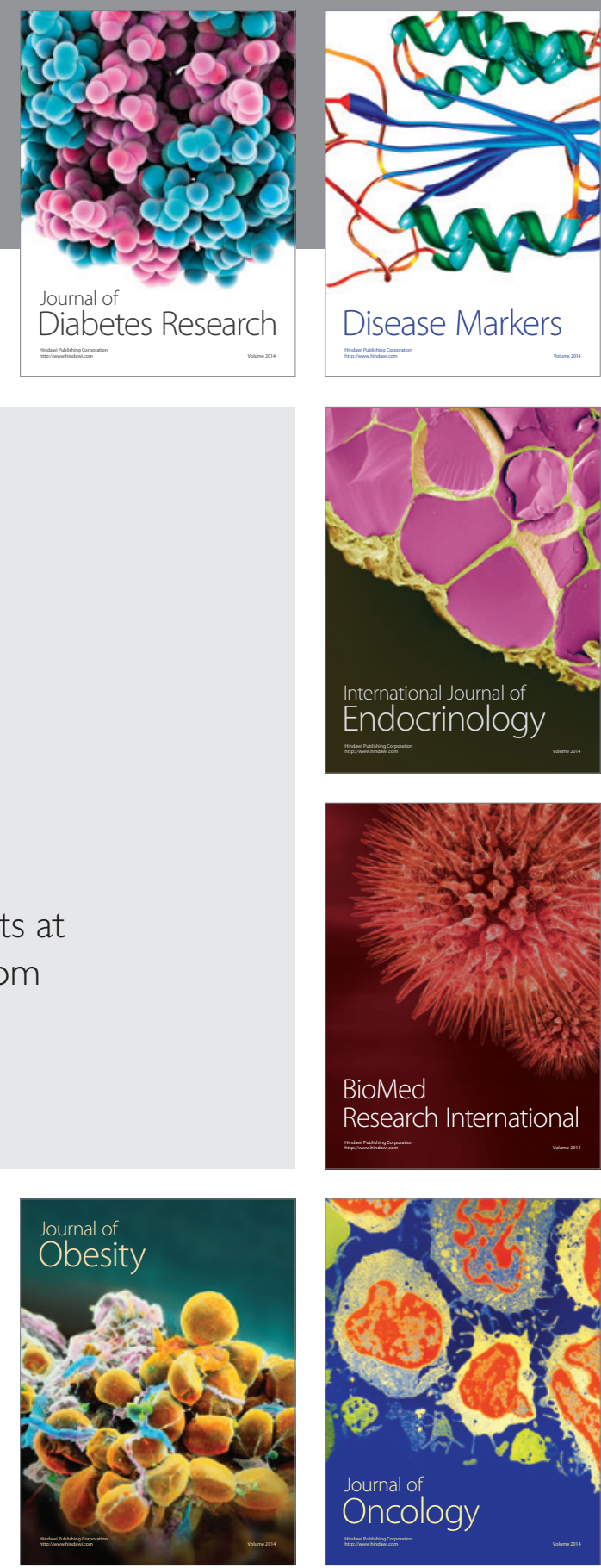

Disease Markers
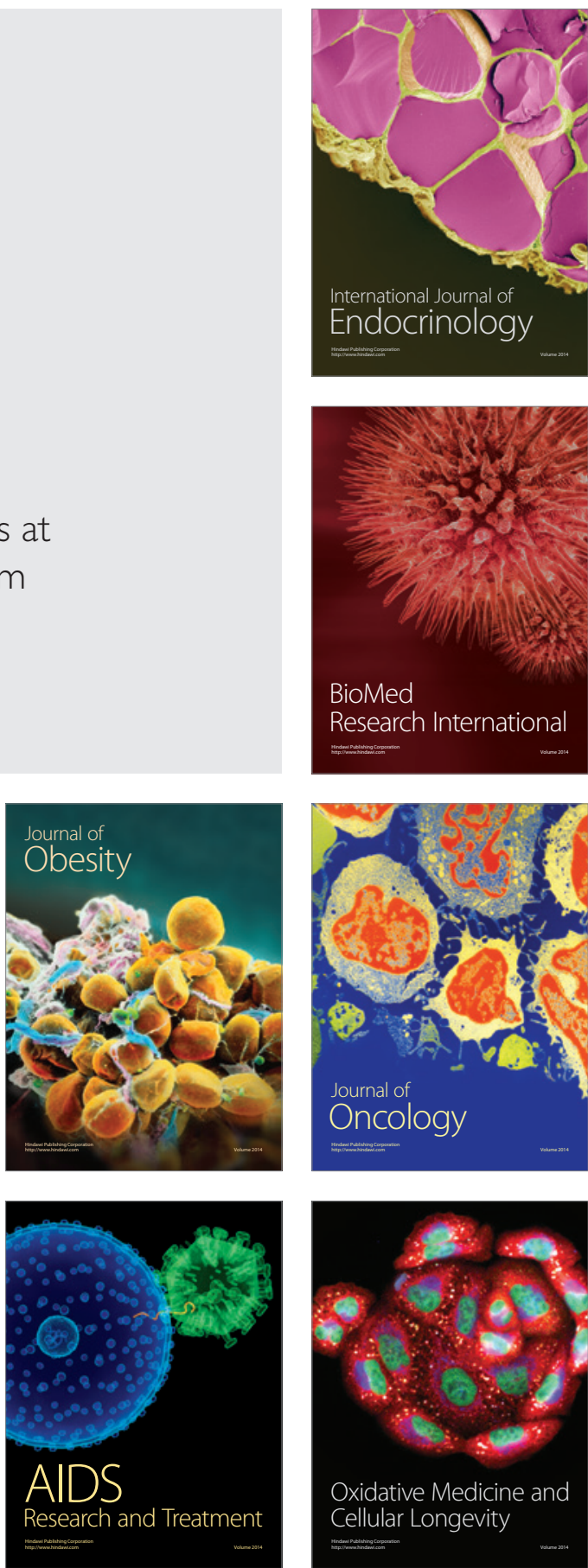